\title{
Testing the hadro-quarkonium model on the lattice
}

\author{
Francesco Knechtli*, Maurizio Alberti \\ Department of Physics, Bergische Universität Wuppertal \\ Gaußstraße 20, 42119 Wuppertal, Germany \\ E-mail: knechtli@physik.uni-wuppertal.de, alberti@uni-wuppertal.de
}

\section{Gunnar S. Bali, Sara Collins, Wolfgang Söldner}

Institut für Theoretische Physik, Universität Regensburg

Universitätsstraße 31, 93053 Regensburg, Germany

E-mail: gunnar.bali@ur.de, sara.collins@ur.de,

wolfgang.soeldner@physik. uni-regensburg.de

\section{Graham Moir}

Department of Applied Mathematics and Theoretical Physics, Centre for Mathematical Sciences University of Cambridge, Wilberforce Road, Cambridge, CB3 OWA, UK

E-mail: graham.moir@damtp.cam.ac.uk

Recently the $\mathrm{LHCb}$ experiment found evidence for the existence of two exotic resonances consisting of $c \bar{c} u u d$ quarks. Among the possible interpretations is the hadro-charmonium model, in which charmonium is bound "within" a light hadron. We test this idea on CLS $N_{\mathrm{f}}=2+1$ lattices using the static formulation for the heavy quarks. We find that the static potential is modified by the presence of a hadron such that it becomes more attractive. The effect is of the order of a few $\mathrm{MeV}$.

WUB/16-09

34th Annual International Symposium on Lattice Field Theory

24-30 July 2016

University of Southampton, UK

\footnotetext{
*Speaker.
} 


\section{Motivation}

The LHCb collaboration analysed the decay $\Lambda_{b} \rightarrow J / \psi p K[1,2]$. A satisfactory description of the data is obtained by adding to the $\Lambda^{*} \rightarrow p K$ resonances two additional resonances of exotic quark content $u u d c \bar{c}$ labeled by $P_{c}^{+}(4380)\left(J^{P}=\frac{3}{2}^{-}\right)$and $P_{c}^{+}(4450)\left(J^{P}=\frac{5}{2}^{+}\right)$and decaying strongly into $J / \psi p$. Flipping the two parities can also explain the data [1,2]. Attractive forces between charmonium and $p p$ systems have been previously conjectured, e.g., to explain the rapid change in the behavior of polarized $p p$ scattering around $\sqrt{s}=5 \mathrm{GeV} \approx m_{p}+m_{p}+m_{J / \psi}$ [3].

Five $(4 q, 1 \bar{q})$ quark systems are very difficult to study directly on the lattice. For example see [4] for a study of a charmonium-nucleon system. Here we test a particular model instead, hadro-quarkonium. In this model quarkonia are bound "within" ordinary hadrons [5]. Examples of charmonium-baryon systems which are close in energy to the LHCb pentaquark candidates are $m(\Delta)+m(J / \psi) \approx 4329 \mathrm{MeV}$ for $J^{P}=\frac{3}{2}^{-}$and $m(N)+m\left(\chi_{c 2}\right) \approx 4496 \mathrm{MeV}$ for $J^{P}=\frac{5}{2}^{+}$.

\section{Hadro-quarkonia in the static limit}

The hadro-quarkonium model can be tested in the static quark limit. To leading order in potential non-relativistic QCD, quarkonia can be approximated by the non-relativistic Schrödinger equation with a static quark-antiquark potential $V_{0}(r)$. The question we want to answer in our study [6] is whether the static potential becomes more or less attractive, when light hadrons are "added". For this we create a zero-momentum projected hadronic state $|H\rangle$ at the time 0 . We let it propagate for an interval $\delta t$ and we then create a quark-antiquark "string". They propagate together for a time interval $t$. We destroy the string at time $t+\delta t$ and finally the light hadron at time $t+2 \delta t$. We compute the correlator

$$
C_{H}(r, \delta t, t)=\frac{\left\langle W(r, t) C_{H, 2 \mathrm{pt}}(t+2 \delta t)\right\rangle}{\langle W(r, t)\rangle\left\langle C_{H, 2 \mathrm{pt}}(t+2 \delta t)\right\rangle},
$$

where we average over the spatial positions of the Wilson loop $W(r, t)$ and over the hadronic sink positions in the hadronic two-point function $C_{H, 2 \mathrm{pt}}$. The difference between the static potential in the presence of a hadron $V_{H}$ and the potential in the vacuum $V_{0}$ can be obtained from

$$
\Delta V_{H}(r, \delta t) \equiv V_{H}(r, \delta t)-V_{0}(r)=-\lim _{t \rightarrow \infty} \frac{\mathrm{d}}{\mathrm{d} t} \ln \left[C_{H}(r, \delta t, t)\right]
$$

and extrapolating $\delta t \rightarrow \infty$.

\section{Lattice results}

We analyse the $N_{\mathrm{f}}=2+1 \mathrm{CLS}$ ensemble "C101" which has $96 \times 48^{3}$ sites, $m_{\pi}=220 \mathrm{MeV}$, $m_{\mathrm{K}}=470 \mathrm{MeV}, L m_{\pi} \approx 4.6, L \approx 4.1 \mathrm{fm}, t_{0} / a^{2}=2.9085(51)$ [7]. It has been simulated using the publicly available openQCD package [8]. The lattice spacing $a=0.0854(15) \mathrm{fm}$ is determined from the scale $\sqrt{8 t_{0}} / a$ [9] extrapolated to physical point [10] and using $\sqrt{8 t_{0}}=0.4144(59)(37) \mathrm{fm}$ [11]. We perform a large statistics calculation consisting of 1552 configurations separated by 4 MDUs, times 12 hadron sources (providing 10 forward and backward propagating two-point functions and for the two sources closest to the open temporal boundaries a forward and a backward 


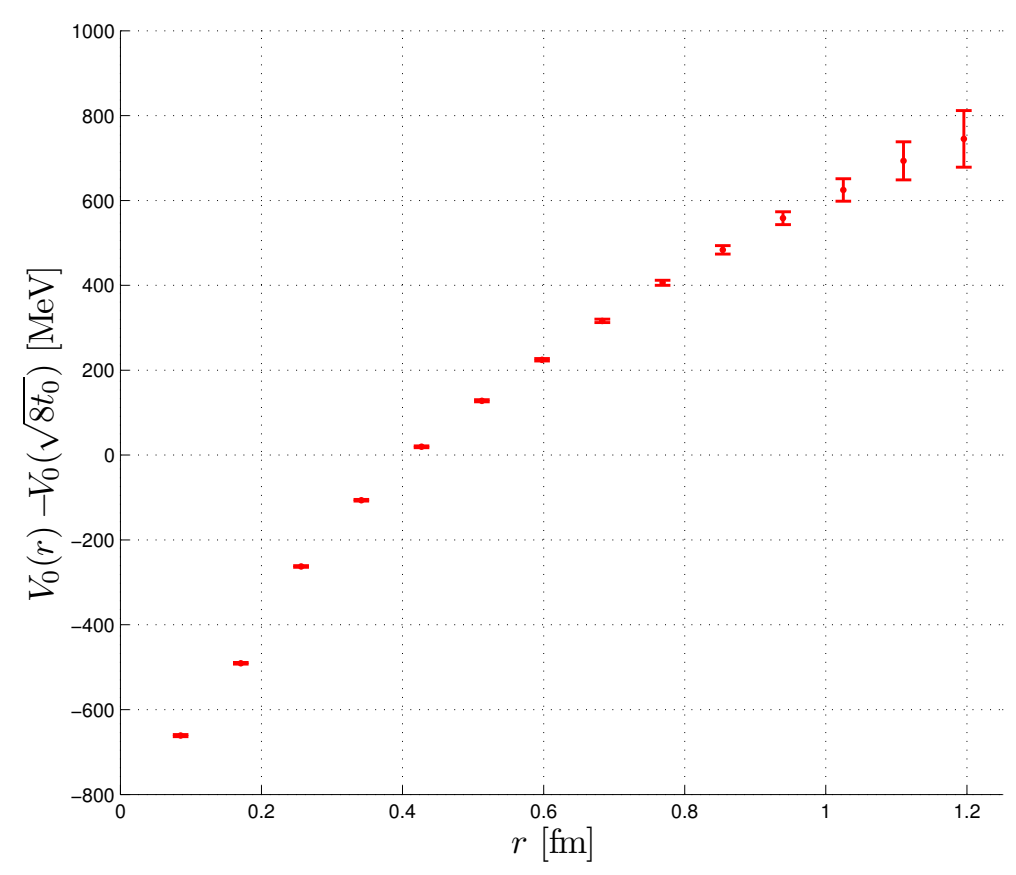

Figure 1: The static potential in the vacuum measured on the CLS ensemble "C101".

two-point function for a total of 22 correlation functions). Wilson loops are measured at all positions and in each direction separately. Hadronic two-point functions and Wilson loops are smeared to optimize their overlap with the respective ground states. We measure $\Delta V_{H}$ for $\pi, K, \rho, K^{\star}$ and $\phi$ mesons; for $N, \Sigma, \Lambda$ and $\Xi$ octet baryons with $J^{P}=\frac{1}{2}^{ \pm}$; and for $\Delta, \Sigma^{\star}, \Xi^{\star}$ and $\Omega$ decuplet baryons with $J^{P}=\frac{3}{2}^{ \pm}$.

We begin the presentation of the results by showing in Fig. 1 the static potential $V_{0}(r)$ in the vacuum. It has been determined using the methods of [12]. We plot $V_{0}(r)$ for distances $r \leq 1.2 \mathrm{fm}$ below the string breaking region.

In order to extract the energy difference $\Delta V_{H}$ in Eq. (2.2) for a given hadron labeled by $H\left(J^{P}\right)$, for each combination of $r$ and $\delta t$, we perform linear fits in $t$ to $\ln \left[C_{H}(r, \delta t, t)\right]$. The range of $t$ for the fits is chosen in the region where the effective energy $a^{-1} \ln \left[C_{H}(r, \delta t, t) / C_{H}(r, \delta t, t+a)\right]$ exhibits a clear plateau. In Fig. 2, Fig. 3, Fig. 4 and Fig. 5 we show the results for the positive parity nucleon, $\Delta$ and $\Sigma^{*}$ as well as for the negative parity $\Sigma^{*}$, respectively. Notice that different colors in the plots correspond to different values of $\delta t$ which are slightly displaced horizontally for clarity. We display the statistical errors only. In [6] we also give estimates of the systematic error by changing the range of the fits.

In Fig. 2 we show $\Delta V_{H}(r, \delta t)$ for the nucleon $N\left(\frac{1}{2}^{+}\right)$. We observe $\Delta V_{H}(r, \delta t)<0$. The results agree for $\delta t \gtrsim 3 a$ and we take the values for $\delta t=5 a$ as good approximation to the limit $\delta t \rightarrow \infty$. The data are well described by a fit to the Cornell parametrization

$$
\Delta V_{H}(r, \delta t=5 a)=\Delta \mu_{H}-\frac{\Delta c_{H}}{r}+\Delta \sigma_{H} r
$$

with the parameters $\Delta \mu_{H}, \Delta c_{H}$ and $\Delta \sigma_{H}$, also shown in Fig. 2. We find that the size of the effect is $\Delta V_{H}(r) \approx-1 \mathrm{MeV}$ to $-2 \mathrm{MeV}$ at a distance $r \simeq 0.3 \mathrm{fm}$ and grows to $\Delta V_{H}(r) \approx-4 \mathrm{MeV}$ to $-7 \mathrm{MeV}$ 


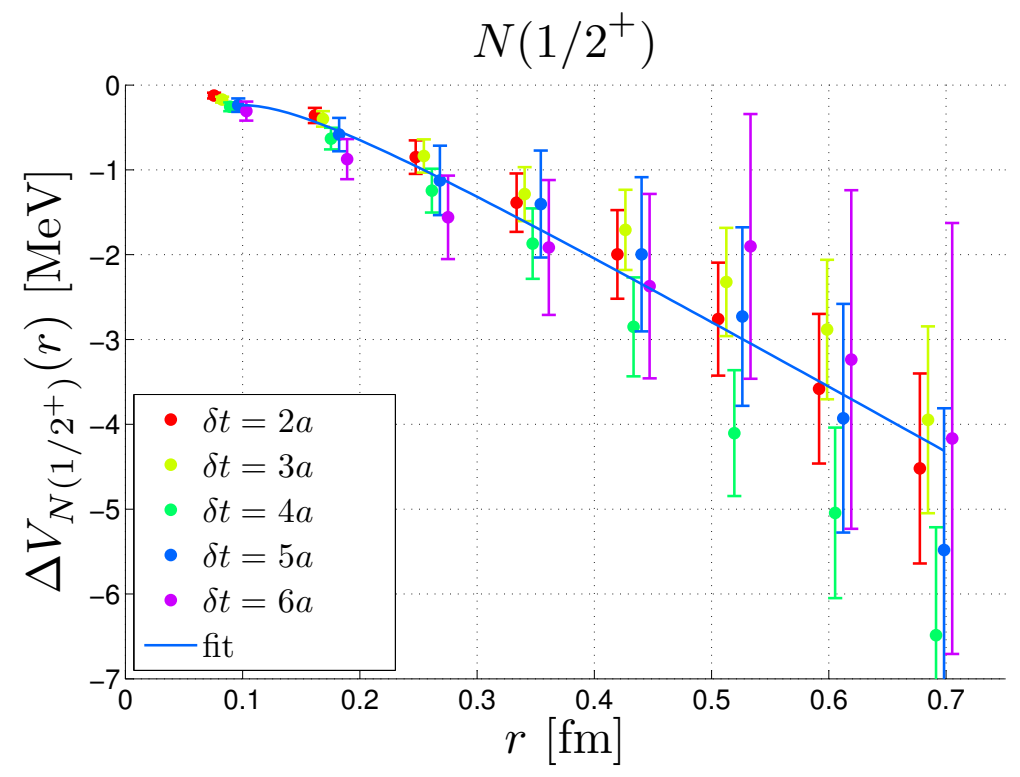

Figure 2: Modification of the static potential "within" a nucleon $N\left(\frac{1}{2}^{+}\right)$.

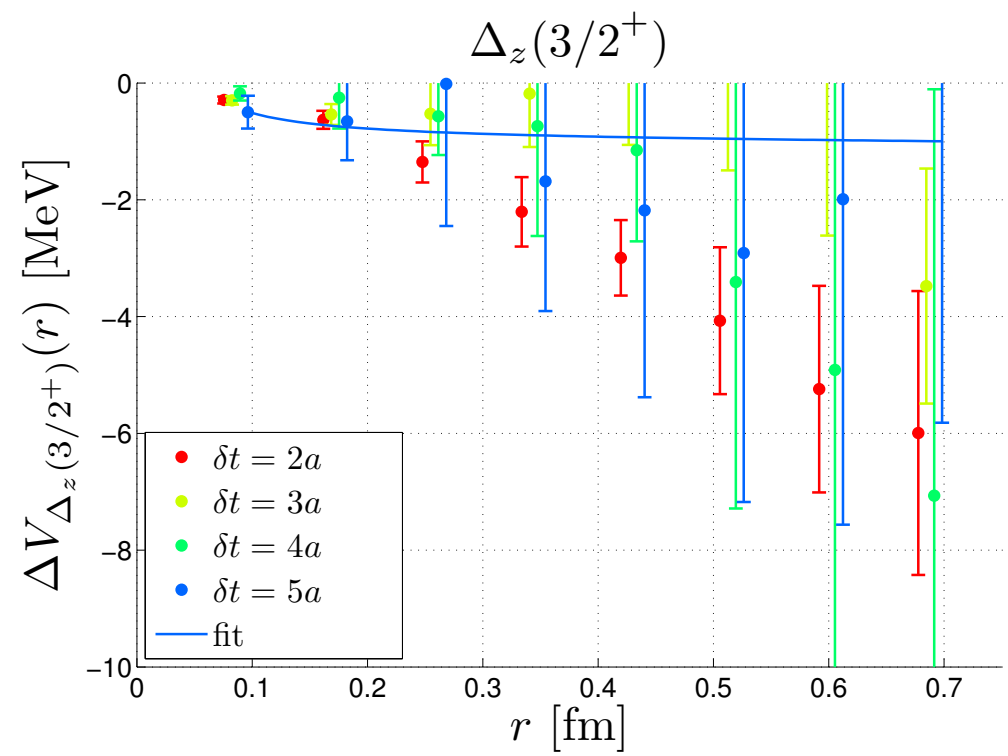

Figure 3: Modification of the static potential "within" a $\Delta\left(\frac{3}{2}^{+}\right)$.

at our largest shown distance $r \simeq 0.7 \mathrm{fm}$. Notice that a bound state of the nucleon $N\left(\frac{1}{2}^{+}\right)$with a $\chi_{c 2}\left(2^{+}\right)$could explain the $J^{P}=\frac{5}{2}^{+}$pentaquark resonance.

In Fig. 3 we show $\Delta V_{H}(r, \delta t)$ for the $\Delta\left(\frac{3}{2}^{+}\right)$. In this case, in Eq. (2.1) we correlate the $\Delta$ polarized in $z$ direction with Wilson loops taken in $z$ direction only, to guarantee that we project onto spin $\Lambda=\left|J_{z}\right|=3 / 2$ along the distance in $z$-direction between the static sources. We find similar results as for the nucleon, albeit with rather large errors. Notice that a bound state of a $\Delta\left(\frac{3}{2}^{+}\right)$with a $J / \psi\left(1^{-}\right)$could explain the $J^{P}=\frac{3}{2}^{-}$pentaquark resonance. As another example with the same spin and parity assignment but with a strange quark content, in Fig. 4 we show $\Delta V_{H}(r, \delta t)$ 


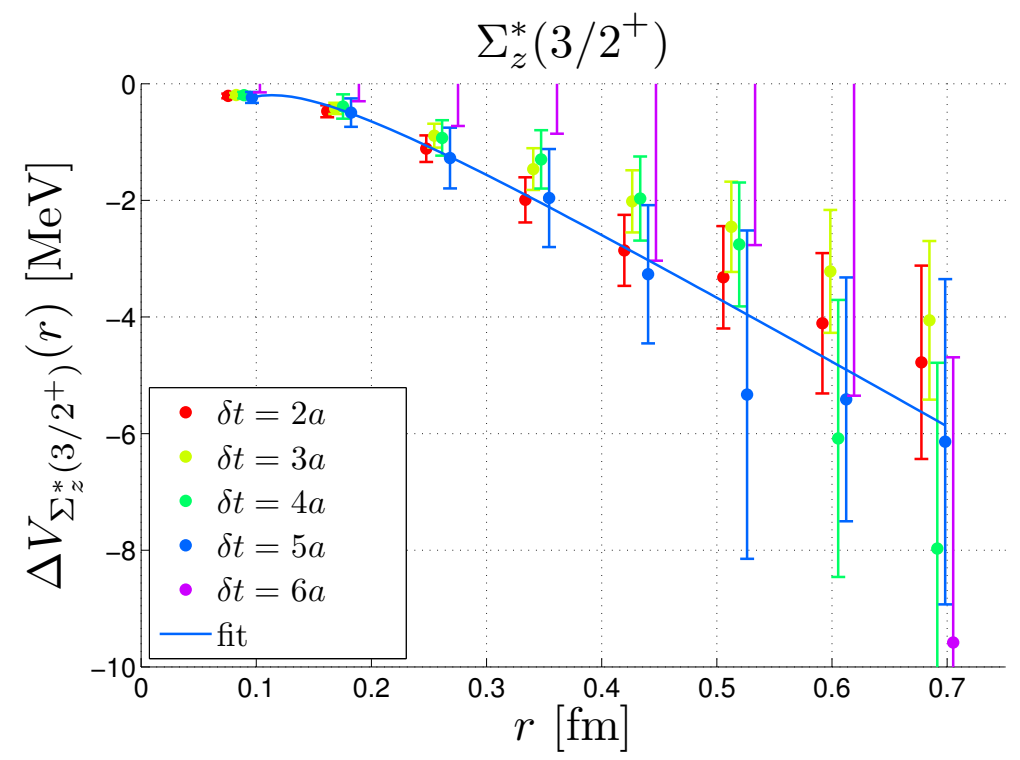

Figure 4: Modification of the static potential "within" a $\Sigma^{*}\left(\frac{3}{2}^{+}\right)$.

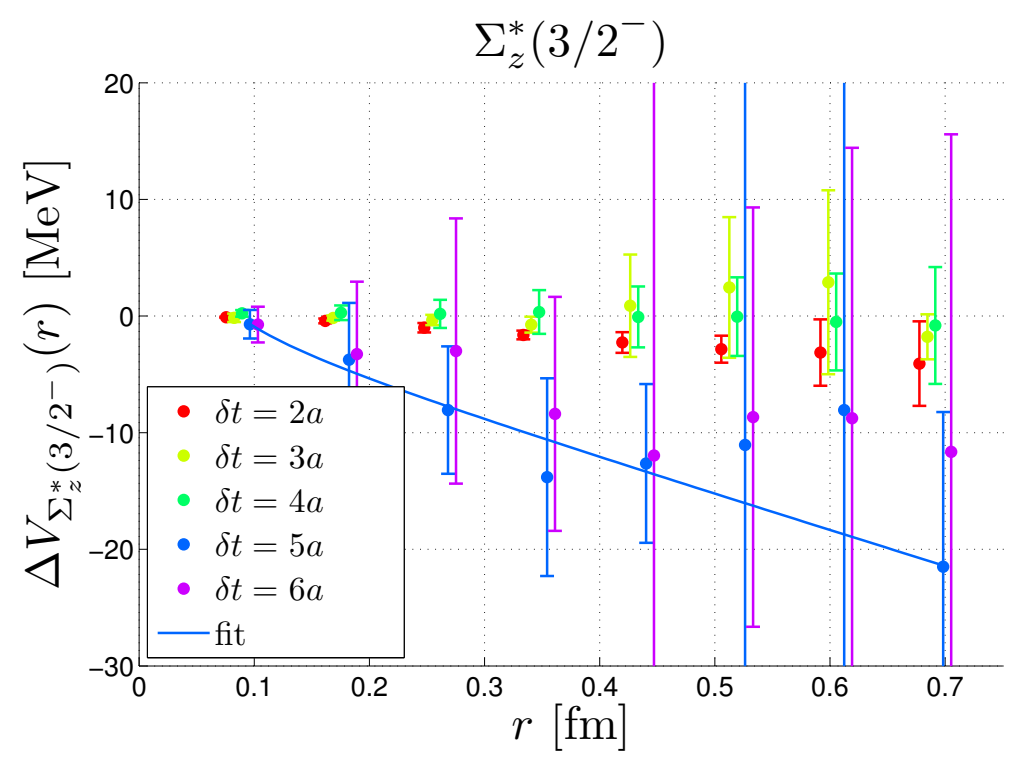

Figure 5: Modification of the static potential "within" a $\Sigma^{*}\left(\frac{3}{2}^{-}\right)$.

for the decuplet $\Sigma^{*}\left(\frac{3}{2}^{+}\right)$. The results are very similar to those for the nucleon.

In Fig. 5 we show an example of $\Delta V_{H}(r, \delta t)$ for a negative parity state, the decuplet $\Sigma^{*}\left(\frac{3}{2}^{-}\right)$. The statistical errors are much larger than for the positive parity case shown in Fig. 4. Within the errors the values of $\Delta V_{H}$ are consistent with the positive parity case but even larger negative values cannot be excluded for the negative parity case. Notice that a bound state of a $\Sigma^{*}\left(\frac{3}{2}{ }^{-}\right)$ with a $J / \psi\left(1^{-}\right)$could give a $J^{P}=\frac{5}{2}^{+}$pentaquark resonance. However in this case it contains a strange quark and also the resulting mass is too large and does not match the mass of the $P_{c}^{+}(4450)$ pentaquark. 


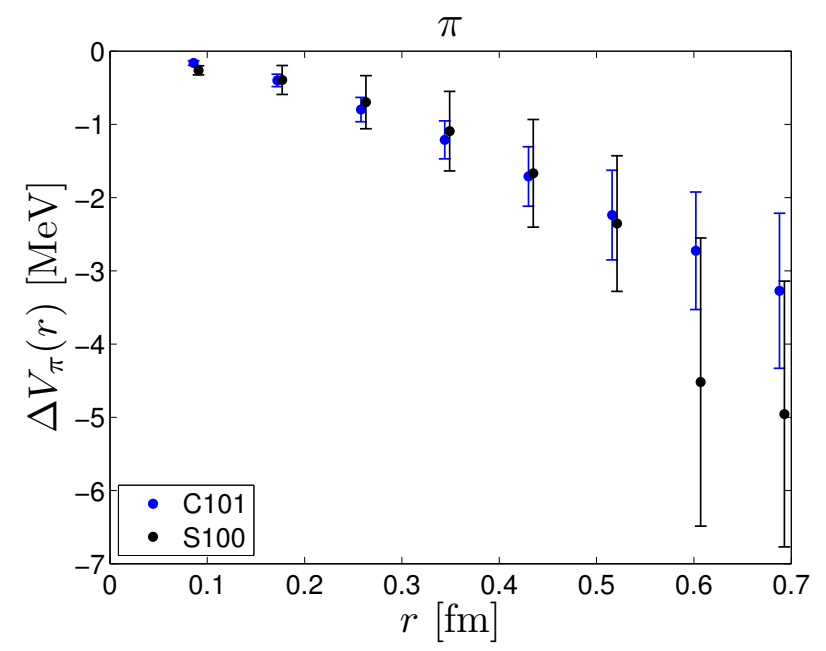

Figure 6: Comparison of the modification of the static potential "within" a pion for two different volumes.

\section{Volume check}

In order to check for finite volume effects we analysed a second CLS ensemble "S100" with a smaller volume of $128 \times 32^{3}$ sites but with the same lattice spacing and quark masses as the ensemble "C101". At the time of the lattice conference the statistics were insufficient to draw a conclusion. By the time these proceedings were written, data for 478 configurations of "S100" were available. In Fig. 6 we compare the results for $\Delta V_{\pi}(r, 5 a)$ on the "C101" and "S100" ensembles and the conclusion is that there are no significant finite volume effects.

\section{Conclusions}

We have numerically established the modification $\Delta V_{H}$ of the static quark-antiquark potential in the presence of a hadron, see Eq. (2.2). We find $\Delta V_{H}(r)<0$. At a distance of $0.5 \mathrm{fm}$ the size of the effect varies between $2 \mathrm{MeV}$ and $3 \mathrm{MeV}$ for all the hadrons we investigated. The main effect can be parametrized as a reduction of the linear slope of the static potential. We emphasize that we do not see finite volume effects, comparing $L m_{\pi} \approx 4.6$ ("C101") with $L m_{\pi} \approx 3.1$ ("S100").

In order to answer the question, whether this modification leads to a larger binding energy of charmonium states, we have compared the energy levels that result from solving the Schrödinger equation with the vacuum static potential $V_{0}$ and with the modified potential $V_{0}+\Delta V_{H}$. Details of this calculation can be found in [6]. The result is a stronger binding of charmonium $1 S$ state by $-1 \mathrm{MeV}$ to $-2.5 \mathrm{MeV}$, of $1 P$ state by $-1 \mathrm{MeV}$ to $-5 \mathrm{MeV}$ and of $2 S$ state by $-1 \mathrm{MeV}$ to $-6.5 \mathrm{MeV}$. These binding energies are similarly small in size as in the deuterium system and may be somewhat inconsistent with the original hadro-charmonium picture.

Acknowledgments. This work was supported by the Deutsche Forschungsgemeinschaft Grant No. SFB/TRR 55. GM acknowledges support from the Herchel Smith Fund at the University of Cambridge and the Deutsche Forschungsgemeinschaft (DFG) under contract KN 947/1-2. We acknowledge PRACE for awarding CLS access to resources on Fermi at CINECA Bologna and on SuperMUC at Leibniz Supercomputing Centre Munich. We also acknowledge computer time 
granted on the "Clover" Cluster of the Mainz Helmholtz Institute for the ensemble generation and on the SFB/TRR 55 QPACE 2 Xeon-Phi installation at Regensburg and on the Stromboli cluster in Wuppertal for the measurements. The calculation of hadronic two point-functions is based on the CHROMA [13] software package. Wilson loops are computed using B. Leder's program available at https://github.com/bjoern-leder/wloop/. For the error analysis we applied the method of [14] including the reweighting factors, see [8].

\section{References}

[1] R. Aaij et al. [LHCb Collaboration], Observation of $J / \psi p$ Resonances Consistent with Pentaquark States in $\Lambda_{b}^{0} \rightarrow J / \psi K^{-} p$ Decays, Phys. Rev. Lett. 115 (2015) 072001, [arXiv:1507.03414] .

[2] R. Aaij et al. [LHCb Collaboration], Model-independent evidence for $J / \psi p$ contributions to $\Lambda_{b}^{0} \rightarrow J / \psi p K^{-}$decays, Phys. Rev. Lett. 117 (2016) no.8, 082002, [arXiv:1604 .05708] .

[3] S. J. Brodsky, I. A. Schmidt and G. F. de Teramond, Nuclear Bound Quarkonium, Phys. Rev. Lett. 64 (1990) 1011.

[4] S. R. Beane, E. Chang, S. D. Cohen, W. Detmold, H.-W. Lin, K. Orginos, A. Parreño and M. J. Savage, Quarkonium-Nucleus Bound States from Lattice QCD, Phys. Rev. D 91 (2015) no.11, 114503, [arXiv:1410.7069].

[5] S. Dubynskiy and M. B. Voloshin, Hadro-Charmonium, Phys. Lett. B 666 (2008) 344 [arXiv:0803.2224].

[6] M. Alberti, G. S. Bali, S. Collins, F. Knechtli, G. Moir and W. Söldner, Hadro-quarkonium from Lattice QCD, arXiv:1608.06537.

[7] M. Bruno et al., Simulation of $Q C D$ with $N_{f}=2+1$ flavors of non-perturbatively improved Wilson fermions, JHEP 1502 (2015) 043, [arXiv:1411.3982] .

[8] M. Lüscher and S. Schaefer, Lattice QCD with open boundary conditions and twisted-mass reweighting, Comput. Phys. Commun. 184 (2013) 519, [arXiv: 1206.2809] .

[9] M. Lüscher, Properties and uses of the Wilson flow in lattice QCD, JHEP 1008 (2010) 071, [arXiv:1006.4518].

[10] G. S. Bali et al. [RQCD Collaboration], Lattice simulations with $N_{f}=2+1$ improved Wilson fermions at a fixed strange quark mass, Phys. Rev. D 94 (2016) no.7, 074501, [arXiv:1606.09039] .

[11] S. Borsanyi et al., High-precision scale setting in lattice QCD, JHEP 1209 (2012) 010, [arXiv:1203.4469].

[12] M. Donnellan, F. Knechtli, B. Leder and R. Sommer, Determination of the Static Potential with Dynamical Fermions, Nucl. Phys. B 849 (2011) 45, [arXiv: 1012.3037].

[13] R. G. Edwards et al. [SciDAC and LHPC and UKQCD Collaborations], The Chroma software system for lattice QCD, Nucl. Phys. Proc. Suppl. 140 (2005) 832, [hep-lat/ 0409003 ] .

[14] U. Wolff [ALPHA Collaboration], Monte Carlo errors with less errors, Comput. Phys. Commun. 156 (2004) 143, Erratum: [Comput. Phys. Commun. 176 (2007) 383], [hep-lat / 0306017 ] . 